\title{
Inhibition of development of laser-induced choroidal neovascularization with suppression of infiltration of macrophages in Smad3-null mice
}

\author{
Hiroki Iwanishi ${ }^{1}$, Norihito Fujita ${ }^{1}$, Katsuo Tomoyose ${ }^{1}$, Yuka Okada ${ }^{1}$, Osamu Yamanaka ${ }^{1}$, Kathleen C Flanders ${ }^{2}$ and \\ Shizuya Saika ${ }^{1}$
}

We evaluated the effects of the loss of Smad3 on the development of experimental argon laser-induced choroidal neovascularization (CNV) in mice. An in vitro angiogenesis model was also used to examine the role of transforming growth factor- $\beta 1$ (TGF $\beta 1) / S m a d 3$ signaling in vessel-like tube formation by human umbilical vein endothelial cells (HUVECs). CNV was induced in eyes of 8-12-week-old B6.129-background Smad3-deficient (KO) mice $(n=47)$ and wildtype (WT) mice $(n=47)$ by argon laser irradiation. Results showed that the size of the CNV induced was significantly smaller in KO mice as compared with WT mice at day 14 as revealed by high-resolution angiography with fluorescein isothiocyanate-dextran. Immunohistochemistry and real-time reverse transcription-polymerase chain reaction of RNA extracted from laser-irradiated choroidal tissues were conducted on specimens at specific timepoints. Invasion of macrophages (F4/80+), but not neutrophils (myeloperoxidase+), and appearance of myofibroblasts ( $a$-smooth muscle actin+) were suppressed in laser-irradiated KO tissues. mRNA expression of inflammation-related factors, that is, vascular endothelial growth factor (VEGF), macrophage-chemoattractant protein-1 (MCP-1), interleukin-6 (IL-6) and TGF $\beta 1$ in choroidal tissues was suppressed by the loss of Smad3. We then examined the effects of adding a Smad3 inhibitor, SIS3, or an ALK5 inhibitor, SB431542, on tube formation promoted by TGF $\beta 1$ or VEGF in HUVECs cocultured with fibroblast feeder. Further addition of SIS3 or SB431542 augmented vessel-like tube formation by HUVECs in the presence of TGF $\beta 1$ or VEGF. In conclusion, lack of Smad3 attenuated the growth of laser-induced CNV with suppression of inflammation by macrophages in mice. Because blocking TGF $\beta 1 / \mathrm{Smad} 3$ signal stimulated the activity of angiogenesis of HUVECs in vitro, the reduction of CNV in vivo in KO mice is attributed to a decrease in growth factor levels in the tissue by the loss of Smad3.

Laboratory Investigation (2016) 96, 641-651; doi:10.1038/labinvest.2016.30; published online 7 March 2016

Choroidal neovascularization (CNV) and associated fibrotic tissue formation around $\mathrm{CNV}$ are the major features of the exudative form of age-related macular degeneration (AMD). ${ }^{1,2} \mathrm{CNV}$ can also occur in a highly myopic eye or in an eye following ocular fundus trauma. In the majority of cases, CNV impairs function of macula, leading to severe visual disturbance. Although the exact mechanism of the development of CNV lesion needs to be investigated, involvement of chronic local inflammation against abnormal subretinal deposits is proposed. ${ }^{3}$ Once CNV extends into the space between retinal pigment epithelium and the photoreceptor layer, subretinal plasma exudation or hemorrhage lead to the formation of fibrous scar-like tissue beneath the retina. ${ }^{4}$ Intracameral administration of antivascular endothelial cell growth factor (VEGF) antibody exerts a prominent therapeutic effect on the exudative form of CNV by inhibiting growth of neovascularization and suppression of subretinal plasma exudation. ${ }^{5,6}$ However, there are cases that exhibit lower effectiveness of anti-VEGF antibody therapy. ${ }^{7,8}$ Moreover, it was reported that blocking VEGF could induce fibrotic process in $\mathrm{CNV}$ lesion that further destroy the structure of macula. ${ }^{9}$

Argon laser irradiation induces $\mathrm{CNV}$ in mice or rats and is used as a model of human CNV lesions. ${ }^{10,11}$ In addition to

\footnotetext{
${ }^{1}$ Department of Ophthalmology, Wakayama Medical University School of Medicine, Wakayama, Japan and ${ }^{2}$ Laboratory of Cancer Biology and Genetics, National Cancer Institute, National Institutes of Health, Bethesda, MD, USA

Correspondence: Dr H Iwanishi, MD or Dr S Saika, MD, PhD, Department of Ophthalmology, Wakayama Medical University School of Medicine, 811-1 Kimiidera, Wakayama 641-0012, Japan.

E-mail: hiwanish@heart.ocn.ne.jp or shizuya@wakayama-med.ac.jp

Received 7 April 2015; revised 5 January 2016; accepted 6 January 2016
} 
blocking VEGF signaling, there is an ongoing investigation into non-VEGF components involved in the development of CNV. ${ }^{12-18}$ In such reports, involvement of local inflammation in the development of laser-induced CNV has been seen in animal studies and in clinical samples. ${ }^{19}$ Macrophages are considered to have a critical role in laser-induced or inflammation-related $\mathrm{CNV}$ formation. ${ }^{20-29}$ Mice that lack c-Jun N-terminal kinase 1 (JNK1) also exhibit less inflammatory reaction and suppression of growth of $\mathrm{CNV}$ postlaser irradiation, suggesting that suppression of local tissue inflammation leads to inhibition of neovascularization in tissue. $^{30}$

We previously reported that loss of or blocking transforming growth factor $-\beta(\mathrm{TGF} \beta) / \mathrm{Smad} 3$ signal inhibits inflammatory fibrosis in injured cornea or conjunctiva in mice. ${ }^{31-36}$ TGF $\beta$ uses the ALK5 receptor for phosphorylation of Smad3 and ALK1 for activation of Smad1/5/8. The signal activated by the former receptor mediates the antiangiogenic behavior and the signal by the latter exhibits the angiogenesis promotion activity in vascular endothelial cells. ${ }^{37}$ The effect of the loss of Smad3 on laser-induced CNV formation might be affected by the two components, suppression of local inflammation and attenuated development of neovascularization activity in vascular endothelial cells in the absence of Smad3. However, the outcome of the formation of CNV upon laser irradiation in mouse ocular fundus in the inhibition or absence of Smad3 has not yet been examined. In the present study, we therefore investigated the effects of gene deletion of Smad3 on the growth of laser-induced CNV in mice. We show here that the loss of Smad3 attenuates the development of laser-induced $\mathrm{CNV}$ in association with suppression of inflammation and fibrotic reaction in mice, although in vitro blocking of the Smad2/3 signal promotes the formation of vessel-like tube structures of vascular endothelial cells.

\section{MATERIALS AND METHODS}

Experiments were approved by the DNA Recombination Experiment Committee and the Animal Care and Use Committee of Wakayama Medical University. They were conducted in accordance with the ARVO Statement for the Use of Animals in Ophthalmic and Vision Research.

\section{Experimental CNV Model in KO Mice}

Argon laser-induced $\mathrm{CNV}$ was generated by a previously described technique with some modifications. ${ }^{38}$ Male 8-12week-old B6.129-background Smad3-deficient (KO) mice $(n=5)$ and wild-type (WT) mice $(n=5)$ were used for evaluation of the size of CNV. Retina in each eye received laser photocoagulation for the induction of experimental $\mathrm{CNV}$ under a slit-lamp microscope observation and an argon laser photocoagulator. A coverslip was applied to the cornea to view the retina with sodium hyaluronate. Four lesions were produced using a power of $150 \mathrm{~mW}$, a spot size of $80 \mu \mathrm{m}$, and a duration of $0.05 \mathrm{~ms}$. Lesions were located at the 3-, 6-, 9-, and 12-o'clock meridians centered on the optic nerve and were located two or three disc diameters from the optic nerve. Fourteen days after laser photocoagulation, all 40 laser spots were used for fluorescein-dextran angiography to quantify the size of the CNV lesion in each of five WT or five KO mice as follows.

\section{Histology and Immunohistochemistry}

Six KO and six WT mice were used. Ocular fundus laser coagulation was performed as described above. At day 3 $(n=4)$ or day $14(n=2)$, the mice were killed and the eyes were processed for paraffin section or cryosectioning. The paraffin sections $(5 \mu \mathrm{m})$ of day 14 specimens were stained with hematoxylin and eosin (HE). The cryosections $(5 \mu \mathrm{m})$ of day 3 were stained for phospho-Smad2 and phosphoSmad1/5/8 or double immunostained for CD31 and glial fibrillary acidic protein (GFAP). Primary antibodies used were: rabbit polyclonal anti-phospho-Smad2 (Ser465/467) antibody (1:100 in PBS; Millipore, Bedford, MA, USA), goat polyclonal anti-phospho-Smad1/5/8 (Ser463/465) antibody (1:100 in PBS; Santa Cruz Biotechnology, Santa Cruz, CA, USA), rat monoclonal anti-CD31 antibody (1:100 in PBS; Santa Cruz Biotechnology), and rabbit polyclonal anti-GFAP antibody (1:500; DAKO, Glostrup, Denmark). After overnight reaction with each primary antibody and subsequent rinsing in PBS, the specimens were treated with secondary antibodies. Antibodies used were: Alexa Fluor 488-conjugated anti-rabbit IgG (H \& L) (1:200 in PBS; Life Technologies Corporation, Carlsbad, CA, USA) for phospho-Smad2 staining, Alexa Fluor 488-conjugated anti-goat IgG (H \& L) (1:200 in PBS; Life Technologies Corporation) for phospho-Smad1/5/8 staining, FITC-conjugated anti-goat IgG (1:100 in PBS; Cappel, MP Biomedicals, LLC, Solon, OH, USA) for GFAP immunostaining, and rhodamine-conjugated anti-rabbit $\operatorname{IgG}(1: 100$ in PBS; Cappel) for CD31 staining. All samples were mounted (VECTASHIELD Mounting Medium with DAPI; Vector Lab, Burlingame, CA, USA). Sections were observed under a confocal microscope (Apotome 2; Carl Zeiss, Jena, Germany).

\section{Quantitation of the Size of CNV Lesions}

We evaluated the size of CNV by using angiography in choroidal flat mounts as previously reported. ${ }^{38}$ Fourteen days after laser photocoagulation, mice were anesthetized and perfused through the left ventricle with a 26-gauge cannula with $1 \mathrm{ml}$ of $50 \mathrm{mg} / \mathrm{ml}$ fluorescein isothiocyanate (FITC)labeled dextran in $10 \%$ gelatin $\left(2 \times 10^{6} \mathrm{MW}\right.$; Sigma, St Louis, $\mathrm{MO})$. The mice were killed by $\mathrm{CO}_{2}$ asphyxiation. The eyes were enucleated and fixed in $4 \%$ paraformaldehyde for $1 \mathrm{~h}$. The anterior segment, crystalline lens, and retina were removed from the eyeball. The remaining sclera, with choroid and retinal pigment epithelium (RPE), was flat-mounted after several relaxing radial incisions and cover-glassed. Specimens were analyzed by fluorescence microscopy (U-CMAD3; Olympus, Tokyo, Japan) in a masked fashion by a research assistant. WinROOF software (Mitani Corporation, Tokyo, Japan) was used to measure the size of the hyperfluorescent 
areas corresponding to CNV. A two-sample Student's t-test with unequal variance was used for statistical analyses of the quantitative CNV size in flat-mounted specimens.

\section{Immunohistochemistry in Flat-Mounted Choroidal Tissue Specimens}

Six KO and six WT mice were used. At $3(n=3)$ or $35(n=3)$ days after laser treatment, cornea and crystalline lens were removed from the enucleated eye, followed by preparing a radial relaxing incision in the eye cup of choroid and sclera. The tissues were then fixed with $4 \%$ paraformaldehyde for $20 \mathrm{~min}$ and the retina and sclera were removed from the choroid of the eye cup. Flat mount specimens of choroidal tissue obtained at day 3 or 35 was immunostained for F4/80 and myeloperoxidase (MPO) or $\alpha$-smooth muscle actin $(\alpha S M A)$, respectively. The samples were incubated with rat monoclonal anti macrophage F4/80 antibody (Clone A3-1, 1:50 dilution in PBS; BMA Biomedicals, August, Switzerland), rabbit polyclonal anti-myeloperixidase (MPO) antibody (1:100 dilution in PBS; NeoMarkers, Fremont, CA, USA) antibody or mouse monoclonal anti- $\alpha$ SMA antibody (1:100 dilution in PBS; NeoMarkers) at $4{ }^{\circ} \mathrm{C}$ for $24 \mathrm{~h}$. The specimens were then incubated for $2 \mathrm{~h}$ at room temperature with FITC-conjugated anti-goat IgG (1:100 dilution; Cappel) for F4/80 or $\alpha$ SMA and rhodamine-conjugated anti-rabbit $\operatorname{IgG}(1: 100$ dilution; Cappel) for MPO after being washed in phosphate-buffered saline (PBS). All samples were mounted (Aqua-Poly/Mount; Funakoshi, Tokyo, Japan). Photomicrographs were captured with a digital camera mounted on a fluorescence microscope (ECLIPS E800; Nikon, Tokyo, Japan).

\section{Real-Time Reverse Transcription-Polymerase Chain Reaction}

For the purpose of understanding the effects of lack of or blocking Smad3 on angiogenic gene expression and inflammation, we performed gene expression analysis in the laser-irradiated eyes by using real-time reverse transcriptionpolymerase chain reaction (RT-PCR). We used both eyes of 20 adult WT or $20 \mathrm{KO}$ mice for laser irradiation. Retina/ choroid in each eye received laser photocoagulation for the induction of experimental CNV under slit-lamp microscope observation as described above. Fifteen laser spots were prepared in each eye. At day 1 (20 eyes in each genotype of mice) and day 3 (20 eyes in each genotype of mice), the mice were killed by $\mathrm{CO}_{2}$ asphyxia. Each eye was enucleated. The cornea, iris, lens, and retina were removed from the sclera, and the tissue of RPE-choroid-sclera complex was obtained. Four tissue samples of RPE-choroid-sclera complex were merged into one RNA sample; thus, we had five RNA samples in each experimental condition, as reported previously. ${ }^{39,40}$ The expression level of TGF $\beta 1$, MPO, F4/80, MCP-1), IL-6, VEGF, and $\alpha \mathrm{SMA}$ were assayed with real-time RT-PCR (TaqMan; Propriety Gene Expression Assays; Applied Biosystems, Foster City, CA). (As for examination of TGF $\beta 1$ mRNA expression 20 eyes at day 1 in each genotype of mice and 40 eyes at day 3 in each genotype of mice were used for RNA extraction.) All the data were normalized for endogenous expression of glyceraldehyde-3-phosphate dehydrogenase by using $\Delta \Delta \mathrm{C}_{\mathrm{t}}$ and were statistically analyzed with the MannWhitney $U$-test.

\section{In Vitro Coculture Experiment}

A commercial coculture system of human umbilical vascular endothelial cells (HUVECs) and fibroblasts (NV Kit KZ-2000; Kurabo, Tokyo, Japan) was used to examine effects of cytokines on tube-like structure formation. We examined the effect of SIS3 (1 nM) and SB431542 (10 $\mu \mathrm{M})$ on TGF $\beta 1$ $(1 \mathrm{ng} / \mathrm{ml})$ and VEGF-A $(10 \mathrm{ng} / \mathrm{ml})$ stimulation of vessel-like tube formation according to the protocol provided by the manufacturer. Specificity of the inhibitory effect of SB431542 on Smad2/3 and SIS3 on Smad3 signaling were established. ${ }^{41,42}$ HUVECs were seeded on confluent fibroblast feeder layer in a 24-well culture plate. Eight wells were prepared for each culture condition. Vessel-like tube tissue formed by HUVECs was detected by immunostaining with anti-CD31, an endothelium marker at day 11 of culture. Immunoreactivity was visualized by $3,3^{\prime}$-diaminobenzidine color reaction. Total length of the CD31-labeled structure and the number of branching (bifurcation) were determined in four independent area in each well by a software of the kit manufacturer (KSW 5000U; Kurabo, Tokyo, Japan), and statistically analyzed by using Mann-Whitney $U$-test. Experiments were repeated two times to confirm the reproducibility.

\section{Cytoplasmic Signaling in HUVECs Treated with Each of the Inhibitors}

To examine if the tube-like structure formation by HUVECs treated with each of the inhibitors is associated with the activation/inactivation of mitogen-activated protein kinases (Erk1/2, p38, and JNK). We then ran western blotting of HUVEC samples treated with TGF $\beta 1$ in the presence or absence of either SB431542 or SIS3. The cells (KE-4109; Kurabo, Tokyo, Japan) were cultured until confluency in a $60-\mathrm{mm}$ culture dish in the medium of KE-2150 S (Kurabo) supplemented with a mixture of cell growth assistance (KE-6150; Kurabo) containing fetal bovine serum, epidermal growth factor, hydrocortisone, heparin, gentamycin, and amphotericin B. The cells were further incubated in a medium free from serum and growth factors (KE-2350 S; Kurabo) for additional $12 \mathrm{~h}$ in the presence or absence of either SB43154 $(10 \mu \mathrm{M})$ or SIS3 $(1 \mathrm{nM})$. The cells were then treated with TGF $\beta 1(1 \mathrm{ng} / \mathrm{ml})$ for $0.5,1,2,3$ and $6 \mathrm{~h}$ and were harvested in cell lysis buffer (Sigma) for western blotting. The expression level of phospho-Erk1/2/Erk1/2, phospho-p38/ p38, phospho-JNK/JNK, or phospho-Smad1/5/8/Smad1/5/8 were semiquantified by western blotting as reported previously. ${ }^{43}$ In brief, the cells were harvested in Sigma mammalian cell lysis buffer (100 $\mu \mathrm{l}$ per dish) and processed for SDS-polyacrylamide gel electrophoresis and western 
blotting. Antibodies used were as follows: Erk1/2 (1:1000; Santa Cruz Biotechnology), phospho-Erk1/2 (1:2000; Cell Signaling, Boston, MA, USA), p38 MAPK (1:1000; Cell Signaling), phospho-p38 MAPK (1:1000; Cell Signaling), JNK (1:1000; Santa Cruz Biotechnology), phospho-JNK (1:2000; Cell Signaling), Smad1/5/8 (1:1000; Santa Cruz Biotechnology), and phospho-Smad1/5/8 (1:1000; Santa Cruz Biotechnology). The peak height or area was measured for quantitation (ATTO's Densitometry Software) of each phospho-component and the membrane was then stripped and restained for non-phospho-component. Secondary antibodies used were peroxidase-conjugated polyclonal antibodies (1:2000; DAKO).

\section{RESULTS}

\section{Experimental CNV Model in KO Mice}

Argon laser irradiation successfully induced CNV in WT and KO mice. HE histology showed the presence of scar-like choroidal tissue beneath the damaged retina at the site of laser injury and that the scarring in choroid seems less marked in Smad3 KO mice at day 14 after laser photocoagulation (Figures $1 \mathrm{aA}$ and $\mathrm{B}$ ). Immunohistochemistry also suggested that the growth of CD31-labeled neovascularization in the area of laser irradiation was impaired by the loss of Smad3 (Figures $1 \mathrm{aC}$ and D). GFAP expression, a marker for retinal fibrosis, was also suppressed in the laser-irradiated tissue by the loss of Smad3. a
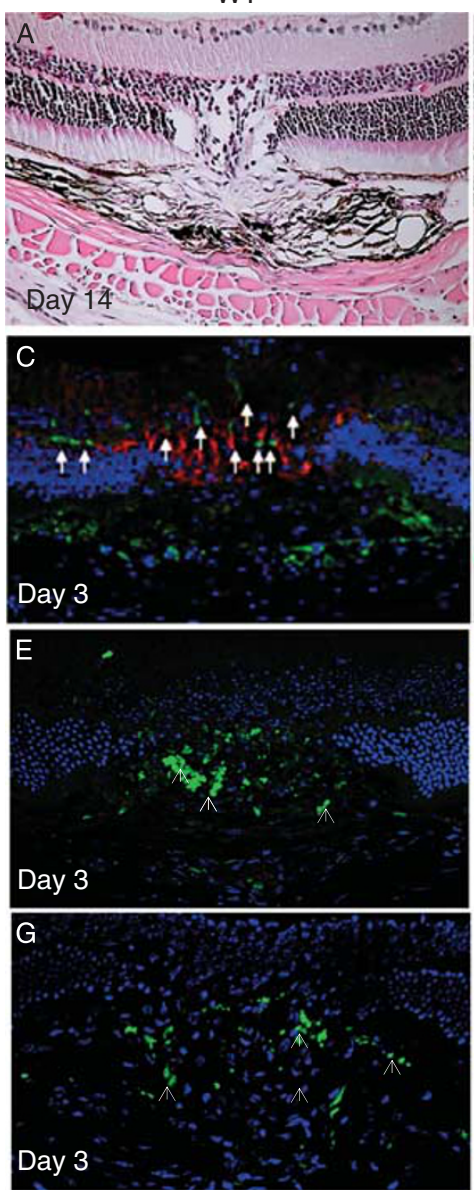
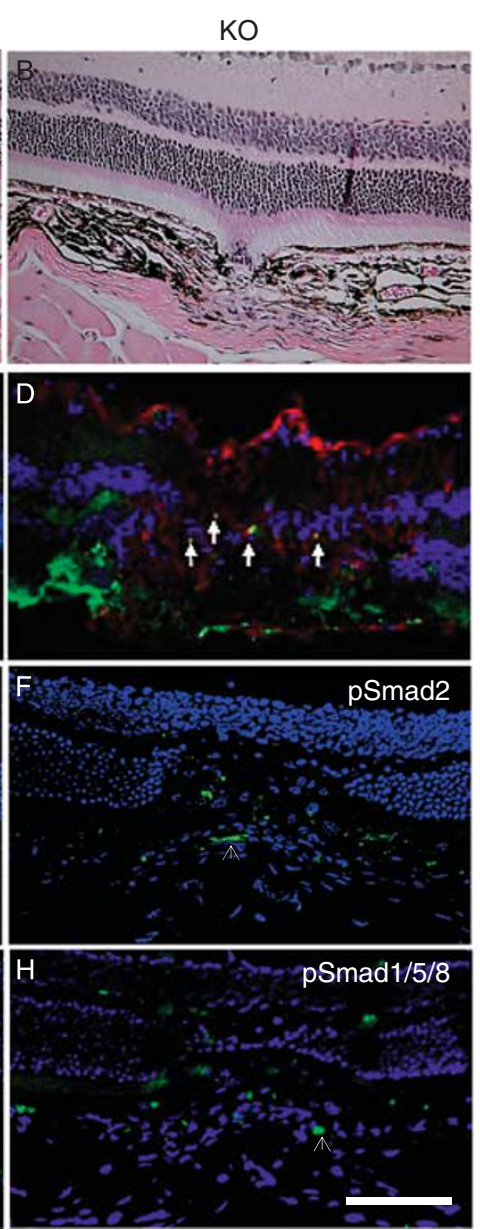

b
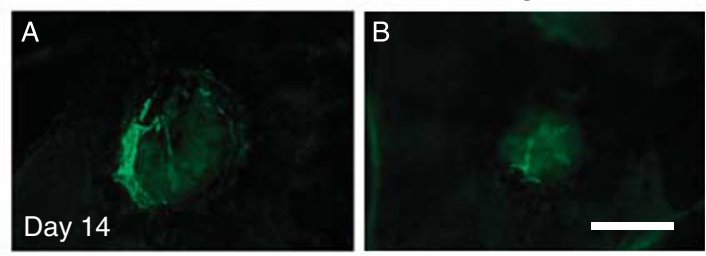

C

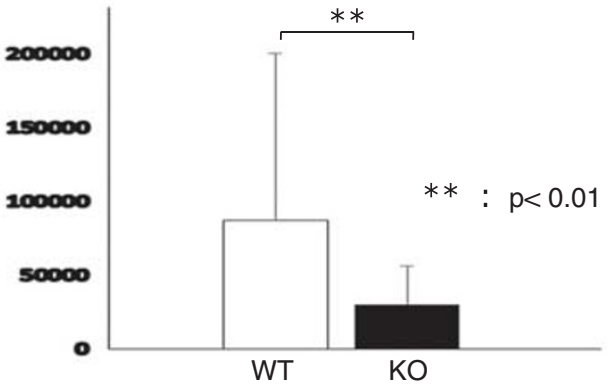

Figure 1 The loss of Smad3 inhibits development of laser-induced choroidal neovascularization (CNV). (a) Histological and immunohistochemical analysis of CNV. Hematoxylin and eosin histology of paraffin sections of the laser-irradiated eye at day 14 indicates destruction of retinal structure and scarring tissue formation in choroid (A and B). Such findings seem more prominent in a wild-type (WT) mouse (A) as compared with a Smad3-null (KO) mouse (B). Expression of Muller glia confirms the damage of the retina by the laser irradiation. Double-immunofluorescence staining for the endothelial cell marker, CD31 (green fluorescein), and glial fibrillary acidic protein (GFAP, rhodamine), a marker for Muller glia activation, in frozen sections obtained of the laser-irradiated eye of a WT mouse (C) and a KO mouse (D) at day 3 after injury. CD31 staining that indicates neovascularization (white arrows) was more frequently observed in a WT mouse at the injured area as compared with a KO tissue. Expression level of phospho-Smad2 (E, F, white arrowheads) and phospho-Smad1/5/8 (G, H, white arrowheads) is inhibited by the loss of Smad3 $(F, H)$ in the injured tissue as compared with WT tissue $(\mathrm{E}, \mathrm{G})$ at day 3. Scale bar, $100 \mu \mathrm{m}$. (b) Angiography with fluorescein isothiocyanate (FITC)-labeled dextran in choroidal flat-mounted specimens show the presence of CNV in both WT and KO tissues (A, B). The size of FITC-labeled CNV seems smaller in a KO choroid (B) as compared with a WT tissue (A). (c) Computer software-assisted analysis shows that the size of CNV is significantly small in KO mice that in WT animals. Y axis indicates total pixels measured. Bars, s.e.m.; ${ }^{* *} P<0.05$ by Student's $t$-test. 
We then examined the activation of Smad2 and Smasd1/5/8 in day 3 tissue specimens. Expression of both phospho-Smad2 and phospho-Smad1/5/8 were reduced by the loss of Smad3 (Figures $1 \mathrm{aE}-\mathrm{H}$ ). Protein expression of both active form and intracellular form of TGF $\beta 1$ by immunohistochemistry with our antibody did not pick up significant staining (data not shown) presumably due to the fact that the ligand level was below the detection by immunostaining.

To quantify the degree of $\mathrm{CNV}$ formation in mice, we evaluated the size of CNV lesions by FITC-dextran perfusion angiography. FITC-dextran perfusion was performed in mice at day 14 after laser photocoagulation and prepared choroidal flat mount specimens after killing mice (Figures $1 \mathrm{bA}$ and $\mathrm{B}$ ). The size of FITC-labeled CNV outgrowths was smaller in the KO group (40 spots in 10 eyes) compared with that in the WT group (40 spots in 10 eyes) (Figure 1c).

\section{Fibrotic Consequence and the Level of Tissue Inflammation in Laser-Irradiated Choroidal Tissue}

We assayed the expression level of F4/80, a macrophage marker, and MPO, a neutrophil marker for the evaluation of the level of tissue inflammation. At day 3, immunostaining for F4/80 and MPO in flat-mounted choroidal specimens suggested that lack of Smad3 suppressed infiltration of macrophages, but not of neutrophils to the laser-injured areas of choroid (Figures $2 \mathrm{a}$ and $\mathrm{b}$ ). At both day 1 and day 3, mRNA expression level of F4/80, but not MPO, in choroidal tissue was significantly suppressed by the loss of Smad3 (Figures 2d and e).

We then used the expression level of $\alpha$ SMA, a marker for myofibroblast, for the evaluation of the degree of tissue fibrosis. At day 35, immunostaining for $\alpha \mathrm{SMA}$ in flat-mounted choroidal specimens suggested that lack of Smad3 suppressed the appearance of myofibroblasts in laser-injured areas (Figure 2c). At day 1, but day 3, mRNA expression level of $\alpha \mathrm{SMA}$ in choroidal tissue was significantly suppressed by the loss of Smad3 (Figure 2f).

Expression of mRNA of VEGF and IL-6 was significantly suppressed in $\mathrm{KO}$ laser-irradiated choroidal tissues as compared with that in the control mice at days 1 and 3 (Figures $3 \mathrm{a}$ and $\mathrm{b}$ ). mRNA expression of TGF $\beta 1$ and MCP-1 was inhibited at day 1 , but not day 3 , in laser-irradiated choroidal tissues by Smad3 deletion as compared with that in WT tissue (Figures $3 \mathrm{c}$ and $\mathrm{d}$ ).

\section{HUVEC Tube Formation Assay}

Adding TGF $\beta 1$ or VEGF to the culture medium significantly promoted the activity of vessel-like tube formation by HUVECs as evaluated by the total length and the number of branching of the CD31-labeled structure (Figure 4). Further, addition of a Smad3 inhibitor, SIS3, significantly augmented the activity of tube formation by HUVECs in the presence of either TGF $\beta 1$ (Figure 4) or VEGF (Figure 4). We then examined if adding an ALK5 inhibitor, SB431542, on the
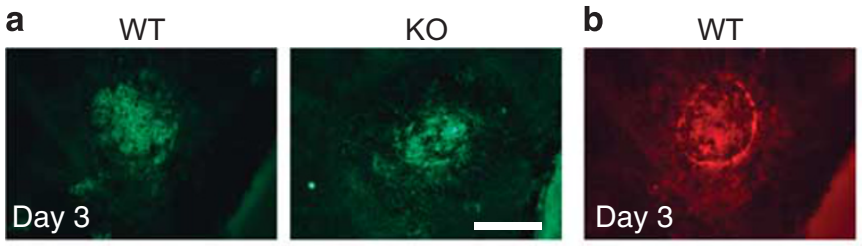

d

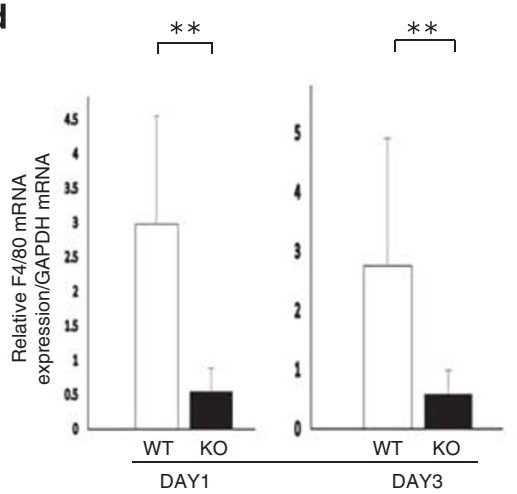

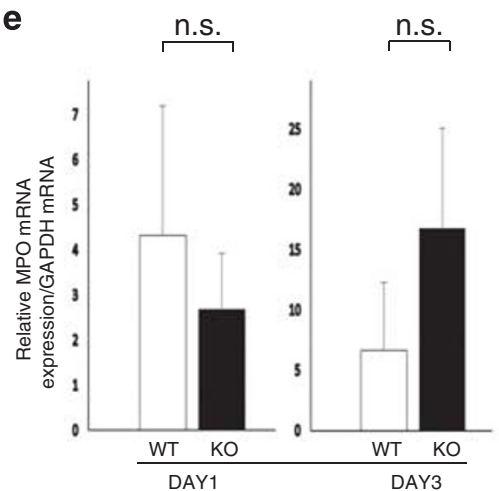
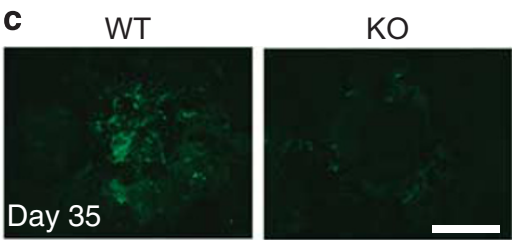

f

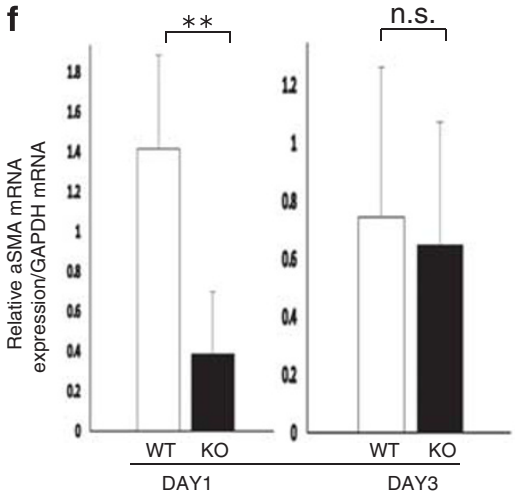

Figure 2 The loss of Smad3 inhibits local tissue inflammation and tissue fibrosis during the development of laser-induced choroidal neovascularization (CNV). Distribution of macrophages (a) and neutrophils (b) are visualized by immunostaining for F4/80 antigen or myeloperoxidase (MPO), respectively, in flat-mounted choroidal tissues at day 3. Appearance of myofibroblasts is detected by immunostaining for $a$-smooth muscle actin ( $a$ SMA) at day 5 (c). Immunohistochemistry suggests that Smad3 deficiency decreases the number of infiltrating macrophages and appearance of myofibroblasts, but not neutrophils, at the area of laser irradiation. Real-time reverse transcription-polymerase chain reaction (RT-PCR) in choroidal (with sclera) samples indicates that expression of F4/80 mRNA (d), but not MPO mRNA (e), is significantly suppressed in a Smd3-null tissue as compared with a wild-type tissue at days 1 and 3. Expression of aSMA mRNA is significantly suppressed in choroidal tissue at day 1, but not at day 3, by Smad3 gene deletion (f). ${ }^{* *} P<0.01$; NS, not significant by by Mann-Whitney $U$-test; bar, mean \pm s.e.m. 

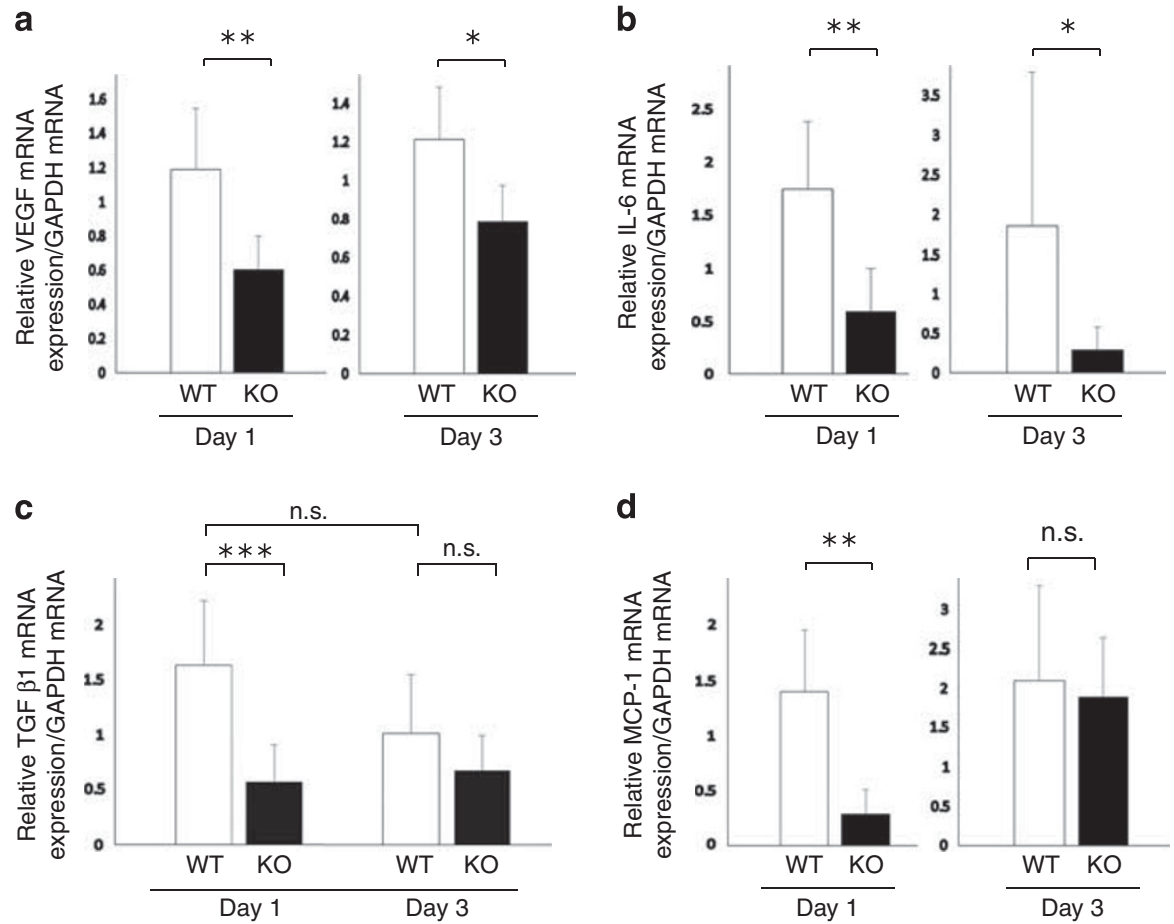

Figure 3 The loss of Smad3 suppresses expression of inflammatory growth factor and cytokines during the development of laser-induced choroidal neovascularization (CNV). mRNA expression level of vascular endothelial growth factor (VEGF) (a), interleukin-6 (IL-6) (b), transforming growth factor $\beta 1$ (TGF $\beta 1)(\mathbf{c})$, and monocyte chemotactic protein-1 (MCP-1) (d) in laser-irradiated tissue samples of retinal pigment epithelium-choroid-sclera complex of WT mice and Smad3-null mice at day 1 and 3 after laser induction. Expression of VEGF and IL- 6 in laser-injured choroidal tissues was suppressed by the loss of Smad3 at days 1 and 3, and that of TGF $\beta 1$ and MCP-1 decreases in Smad3-null tissue at day 1 as compared with wild-type mice. ${ }^{*} P<0.05$, ${ }^{*} P<0.01$; NS, not significant by Mann-Whitney $U$-test; bar, mean \pm s.e.m.

vessel-like tube formation by the cells. Similar to SIS3, adding SB431542 also enhanced the tube formation by the cells in the presence of either TGF $\beta 1$ or VEGF (Figure 5).

\section{Effects of Each Inhibitor on Signal Transduction in HUVECS}

Western blotting was performed on the samples of HUVECs exposed to exogenous TGF $\beta 1$ in the presence or absence of each inhibitor to further understand the effects of each inhibitor on signal transduction in HUVECs. Adding SB431542 potentiated activation of Erk1/2, but not p38, JNK and Smad1/5/8 upon exposure to exogenous TGF $\beta 1$ (Figure 6). On the other hand, treatment of cells with SIS3 did not alter the level of TGF $\beta 1$-induced activation of these four signaling cascades (Figure 6).

\section{DISCUSSION}

In the present study, we show for the first time that the loss of Smad3 reduced laser-induced CNV in mice. Argon laser irradiation of the ocular fundus damages Bruch's membrane, overlaying retina and underlying choroidal tissue and induces inflammation by heat coagulation. Eventually, CNV develops that mimics the human condition wet-type CNV..$^{10,11}$ Human $\mathrm{CNV}$ tissue contains a number of macrophages that are considered to be a major source of angiogenic growth factors in the local tissue. ${ }^{23-27}$
In the present study, examination at day 14 showed that the size of experimental CNV was smaller in a $\mathrm{KO}$ mouse as compared with a WT mouse. Development of CNV is believed to depend on angiogenic growth factors expressed in inflammatory cells that have infiltrated to the laser-injured local tissue. We therefore examined whether lack of Smad3 affects the degree of local tissue inflammation by using immunohistochemistry in flat-mounted choroidal specimens and real-time RT-PCR of RNA samples extracted from laser-injured choroidal tissues. Immunohistochemistry detected accumulation on MPO-labeled neutrophils and F4/80-labeled macrophages in the laser-irradiated areas. Real-time RT-PCR showed that the loss of Smad3 suppressed the expression level of a macrophage marker, F4/80, in irradiated choroidal tissues at days 1 and 3, but did not affect neutrophil infiltration on the tissue. Activation of tissue-deposited TGF $\beta$ by laser injury might not be followed by the further augmentation of inflammation in the absence of Smad3 signal. Expression of VEGF, MCP-1, IL-6, and TGF $\beta 1$ was less prominent in a KO tissue as compared with a WT tissue. Immunohistochemistry showed that the loss of Smad3 suppressed the expression of phospho-Smad2 and phospho-Smad1/5/8 in the laser-irradiated tissue at day 3, suggesting the reduction of the tissue level of specific ligands. Reduction of expression of Smad3-dependent growth factors might further inhibit subsequent invasion of macrophages to the tissue. Reduction 

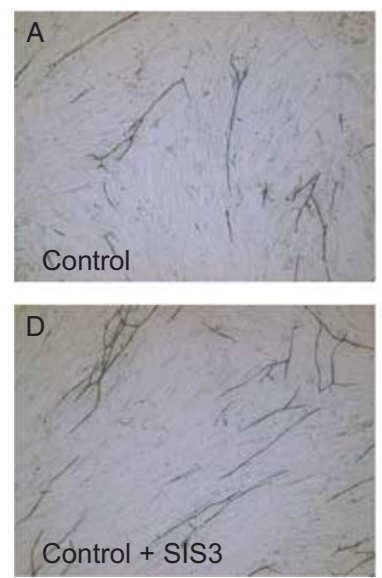
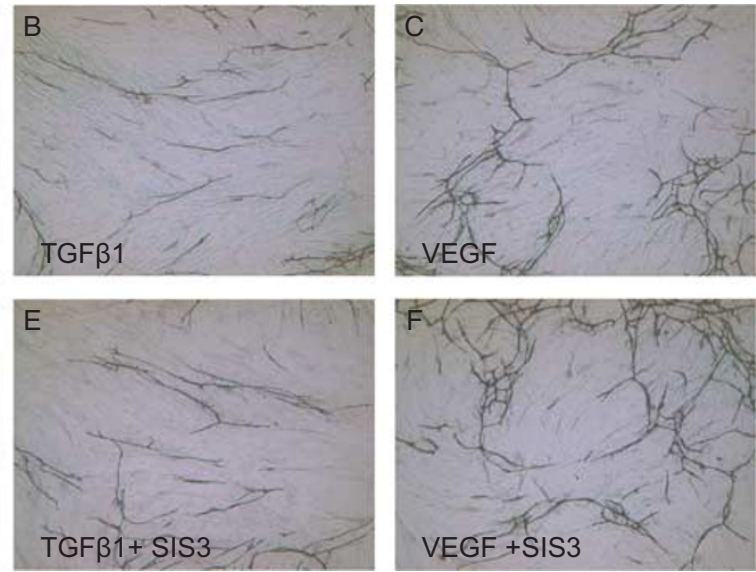

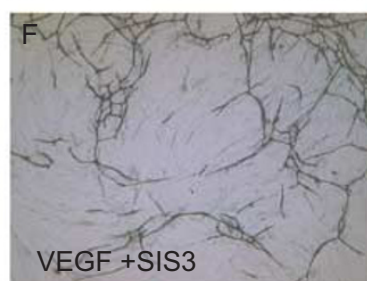

b

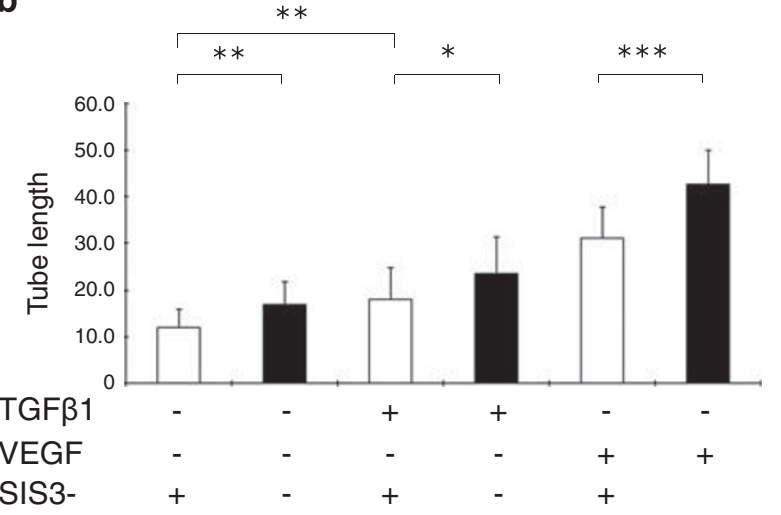

C

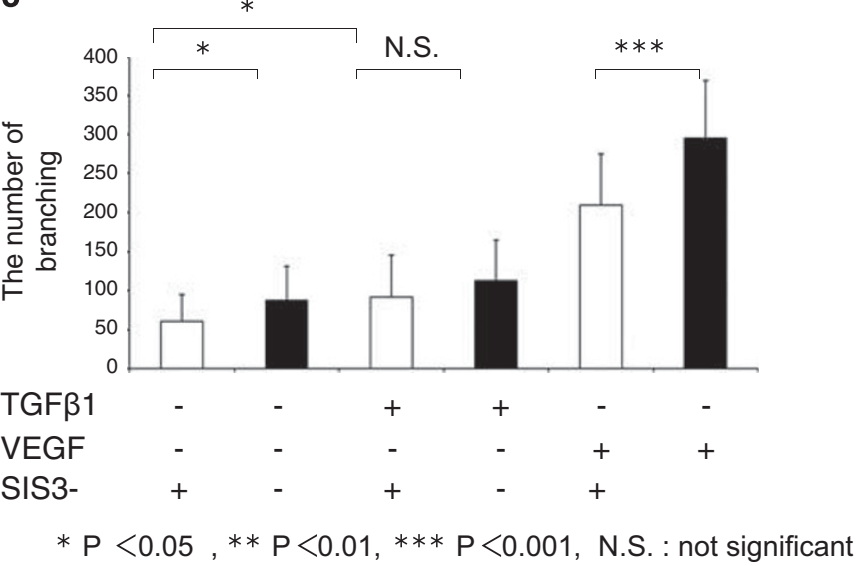

Figure 4 Addition of a Smad3 inhibitor augments vessel-like structure formation by human umbilical vascular endothelial cells (HUVECs) in the presence of transforming growth factor $\beta 1$ (TGF $\beta 1$ ) or vascular endothelial growth factor (VEGF). Vessel-like tube formation by HUVECs is detected by CD31 immunostaining in coculture with fibroblasts in the presence of TGF $\beta 1$ or VEGF. CD31-immunoreactive vessel-like tissue is seen on the fibroblast feeder layer in the control culture without specific ligands (a-A). More dense vessel-like tissue with more frequent branching was seen with $1 \mathrm{ng} / \mathrm{ml}$ TGF $\beta 1$ (a-B) and $10 \mathrm{ng} / \mathrm{ml}$ VEGF-A (a-C). Adding an inhibitor of Smad3 (SIS3) at the concentration of $1 \mathrm{~nm}$ to the culture medium seems to accelerate the formation of tube-like structure by HUVECs (a-D-F). Promotion of tube-like tissue formation by adding SIS3 is confirmed by evaluation of total length of the structure (b) and the number of branching formation (c). ${ }^{*} P<0.05,{ }^{*} P<0.01,{ }^{* * *} P<0.001$ by Mann-Whitney $U$-test; bar, mean \pm s.e.m. N.S.: not significant

of these growth factors or cytokines could be explained by the less invasion of macrophages and was attributed to the attenuation of $\mathrm{CNV}$ growth postlaser treatment by Smad3 gene deletion.

In the clinical setting, fibrosis is also the major feature of wet-type CNV. Recent advances in bioengineering-based therapy has allowed us to treat $\mathrm{CNV}$-related diseases with intracameral administration of an anti-VEGF-neutralizing antibody, which yields satisfactory outcomes in terms of regression of neovascularization and resultant reduction of subretinal or choroidal exudation and visual improvement. However, a remaining issue to be resolved is subretinal/ choroidal tissue fibrosis after regression of neovascularization. $^{44-46}$ In general, the process of tissue fibrosis is mediated by various fibrogenic growth factors including $\mathrm{TGF} \beta$ and connective tissue growth factor (CTGF). CTGF is known to be upregulated by TGF $\beta$ signaling. TGF $\beta$ activates fibrogenic cell behaviors via Smad3 pathway and other noncanonical TGF $\beta$ signals, that is, MAPKs, overall support Smad3-mediated gene responses. We hypothesized that fibrosis associated with laser-induced CNV might also be suppressed by the loss of Smad3 in mice. To explore this question, we analyzed protein and mRNA expression of aSMA by immunohistochemistry and real-time RT-PCR because myofibroblast is the central key player of tissue fibrosis. Immunohistochemistry in flat-mounted choroidal tissue obtained at day 35 suggested that Smad3 deletion inhibited myofibroblast generation in the laser-injured area. ${ }^{47}$ It was also reported that the expression level of GFAP represents the degree of fibrotic reaction of neural retina. ${ }^{48}$ In the current study, protein expression of GFAP in the laser-irradiated neural retina was also suppressed by the loss of Smad3. Real-rime RT-PCR showed suppression of $\alpha \mathrm{SMA}$ mRNA expression at day 1 (but not at day 3 ) in choroidal 

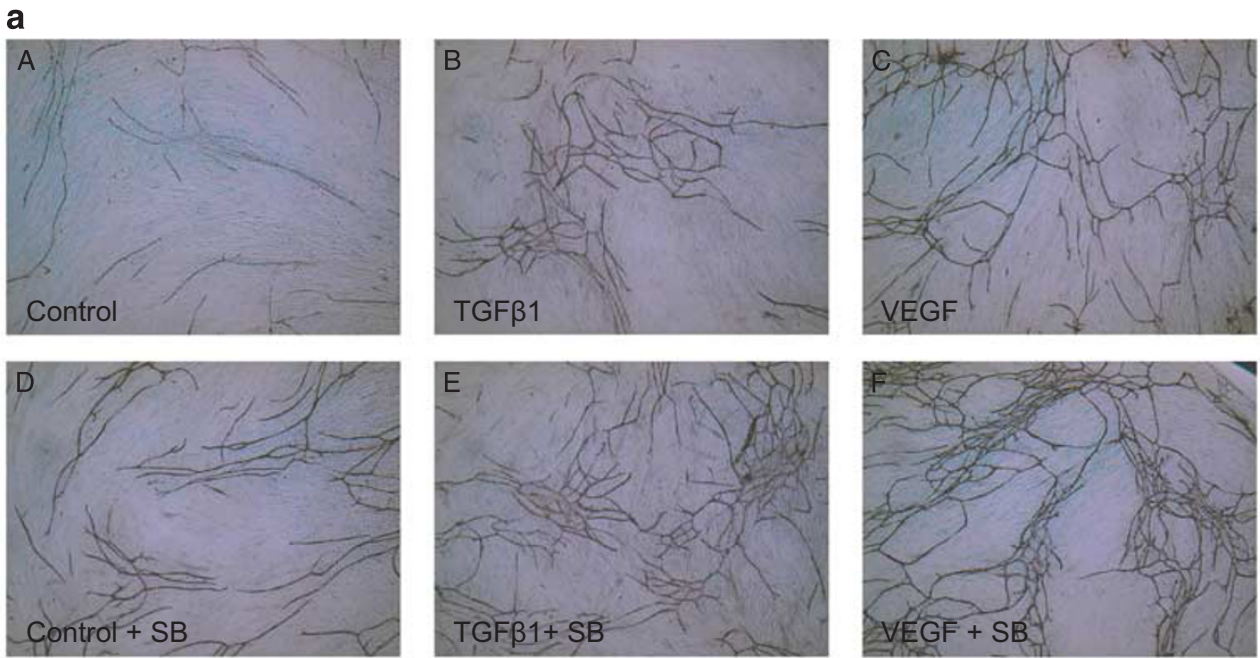

b

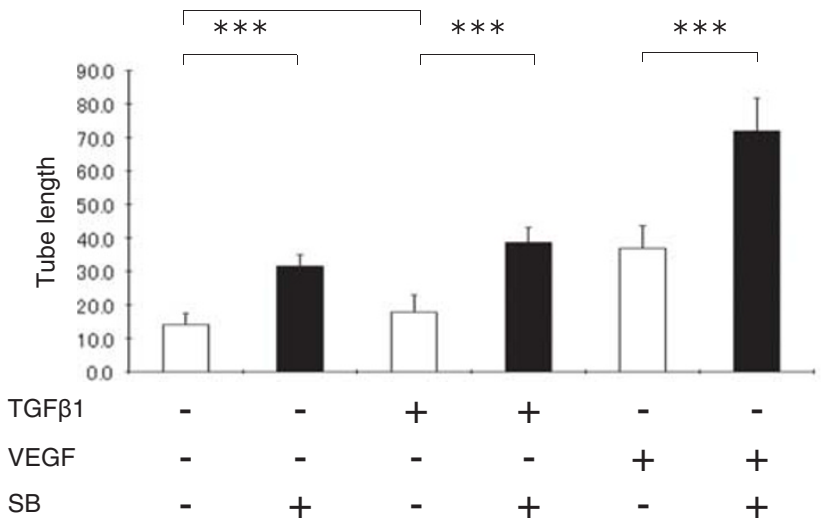

C

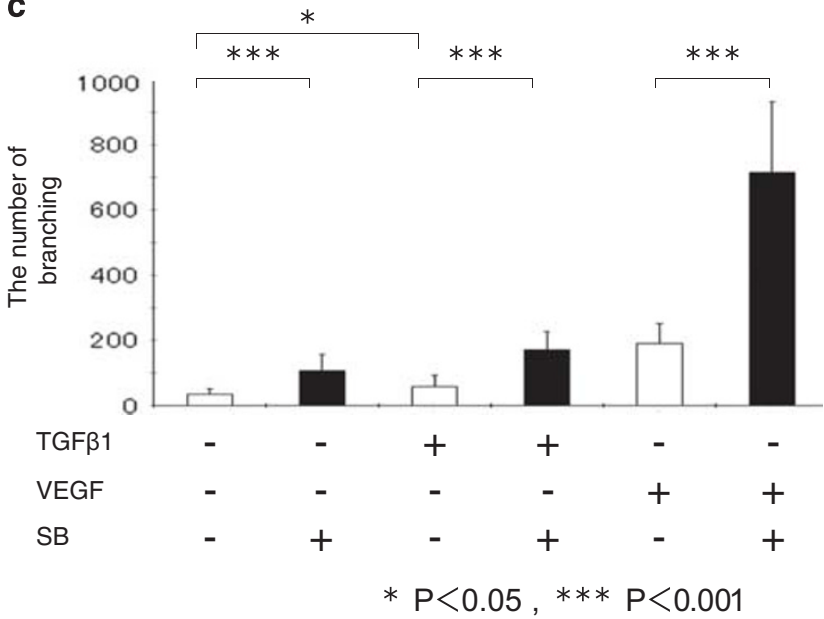

Figure 5 Supplementation of an ALK5 inhibitor enhances vessel-like structure formation by human umbilical vascular endothelial cells (HUVECs) in the presence of transforming growth factor- $\beta 1$ (TGF $\beta 1$ ) or vascular endothelial growth factor (VEGF). Vessel-like tube formation by HUVECs is detected by CD31 immunostaining in coculture with fibroblasts in the presence of TGF $\beta 1$ or VEGF. CD31-immunoreactive vessel-like tissue is seen on the fibroblast feeder layer in the control culture without specific ligands (aA). More dense vessel-like tissue with more frequent branching was seen with $1 \mathrm{ng} / \mathrm{ml}$ TGF $\beta 1(\mathbf{a B})$ and $10 \mathrm{ng} / \mathrm{ml}$ VEGF-A (aC). Adding an inhibitor of ALK5 (SB431542) at the concentration of $10 \mu \mathrm{m}$ to the culture medium seems to accelerate the formation of tube-like structure by HUVECs (aD-F). Promotion of tube-like tissue formation by adding SB431542 is confirmed by evaluation of the total length of the structure (b) and the number of branching formation (c). ${ }^{*} P<0.05,{ }^{* *} P<0.01,{ }^{* * *} P<0.001$ by Mann-Whitney $U$-test; bar, mean \pm s.e.m.

tissue. This finding suggests that blocking Smad3 blocks both new vessel growth and potentially tissue scarring in laser-irradiated choroid.

Recently, Zhandi et al. ${ }^{49}$ reported that macrophage subset conversion has an important role in the development of CNV-type AMD. The M2 subset of macrophages that are involved in containing inflammation and repairing tissue damage, but not M1 macrophages, are reportedly involved in the development of CNV. Infiltration and activation of M2 macrophages are known to be TGF $\beta$-dependent, ${ }^{50,51}$ and thus the current finding that lack of Smad3 attenuates macrophage invasion and development of laser-induced $\mathrm{CNV}$ is consistent with the results of these previous reports. We show here that the loss of Smad3 suppressed local tissue inflammation in choroid postlaser irradiation and attenuated the formation of
$\mathrm{CNV}$ in mice. Decreased macrophage invasion and resultant reduction of the level of angiogenic factors could explain the inhibition of CNV growth. However, the effects of the lack of Smad3 in vascular endothelial behavior also need to be examined to understand the contribution of vascular endothelial cells to the in vivo outcome.

We then examined the effects of blocking Smad3 signaling on the activity of the formation of vessel-like tube structure of HUVECs on fibroblast feeder layer, which mimics in vivo neovascularization activity. It is reported that HUVECs or other vascular endothelial cells, different from other cell types, express two types of TGF $\beta$ receptors, that is, ALK1 and ALK5, that mediate contradicting effect on angiogenesis/ neovascularization. ${ }^{52,53}$ Another study showed that gene transfer of ALK5 caused antiangiogenic activity and inhibited 


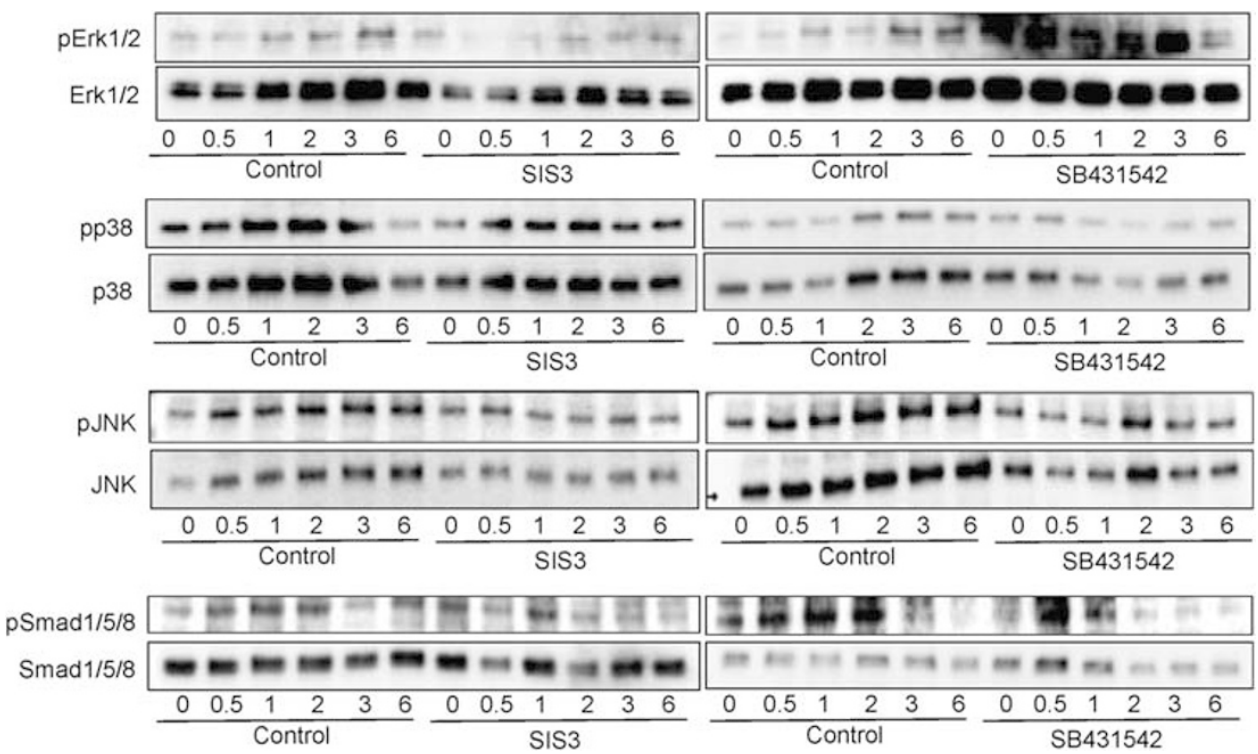

Figure 6 Signal-transduction analysis in human umbilical vein endothelial cells (HUVECs) as examined by western blotting. Confluent cells incubated in serum-free medium and pretreated with either SB431542 or SIS3 as described in the Materials and Methods section. Pretreatment of cells with SIS3 does not significantly affect signaling cascades of Erk1/2, p38, c-Jun N-terminal kinase (JNK), and Smad1/5/8. On the other hand, incubation of the cells with SB431542 upregulates phosphorylation of Erk1/2, but exhibits no significant effects on the signaling of p38, JNK, and Smad1/5/8.

tube formation, whereas ALK1 overexpression did not affect tube formation in HUVECs. ${ }^{54}$ In vascular endothelial cell types, TGF $\beta$ binding to ALK1 mediates signaling for acceleration of cell migration and angiogenesis via upregulation of Id1, whereas TGF $\beta /$ ALK5 complex activates signaling cascades toward inhibition of angiogenesis or new vessel formation. ${ }^{37}$ We show here that adding an inhibitor of Smad3, SIS3 $(1 \mathrm{nM})$, accelerated angiogenic activity of HUVECs cultured on fibroblast feeder layer in the absence or presence of TGF $\beta 1$ or VEGF, indicating that ALK5/Smad3 signal negatively regulated tube formation by HUVECs. Supplementation of SIS3 to the culture medium did not exhibit a significant effect on signaling cascades of Erk1/2, $\mathrm{p} 38$, JNK, and Smad1/5/8, indicating that relative predominance of Smad1/5/8 against Smad3 signal might be much involved in in vitro tube-like structure formation. It was reported, however, that signaling cascades involved in angiogenesis mediated by Smad2 and Smad3 are not equivalent; Smad2, but not Smad3, mediated the expression of soluble VEGF-A receptor sFlt- 1 that is capable of inhibition of VEGF in response to TGF $\beta 1 .{ }^{55}$ Thus, we then blocked the phosphorylation of both Smad2 and Smad3 by adding an ALK5 inhibitor, SB431542, in HUVEC culture on fibroblast feeder, and obtained a result that blocking ALK5 also promoted angiogenic activity of HUVECs similar to the effects of a Smad3 inhibitor SIS3. In general, formation of heterodimer complex of ALK1 and ALK5 is essential to signaling activation by ALK1 receptor upon TGF $\beta$ exposure. ALK1 signal is reportedly abolished in the absence of ALK5 receptor. ${ }^{53}$ However, it was reported that ALK1-endoglin complex is formed in vascular endothelial cells cultured on fibronectin matrix and mediates Smad1/5/8 signal in the absence of ALK5. ${ }^{56}$ In the current experiment, HUVECs were cultured on fibronectin-rich (data not shown) fibroblast feeder later and thus might not require ALK5. The effect of promoting branching of the newly formed tube-like structure was more marked with SB431542 as compared with the samples supplemented with SIS3 in TGF $\beta 1$-plus culture. Erk signal is reportedly involved in in vitro tube-like tissue formation by HUVECs. ${ }^{57,58}$ Although tube formation was clearly accelerated by blocking Smads, blocking ALK5 (thus blocking Smad2/3) might be related to the promotion of formation of branching points via Erk activation. This point remains to be uncovered.

Taken together, the current data show that suppression of macrophage infiltration to the laser-injured local tissue by the loss of Smad3 resulting in the suppression of development of target of treatment of neovascularization and subfoveal fibrosis CNV-related diseases.

\section{ACKNOWLEDGMENTS}

This study was supported by the Grants from the Ministry of Education, Science, Sports, and Culture of Japan (C25462730 (to KT), C24592638 (to YO), C25462759 (to OY), and C19592036 (to SS)).

\section{DISCLOSURE/CONFLICT OF INTEREST}

The authors declare no conflict of interest.

1. Zarbin MA. Current concepts in the pathogenesis of age-related macular degeneration. Arch Ophthalmol 2004;122:598-614.

2. Kent D, Sheridan C. Choroidal neovascularization: a wound healing perspective. Mol Vis 2003;9:747-755. 
3. Crabb JW, Miyagi M, Gu X et al. Drusen proteome analysis: an approach to the etiology of age-related macular degeneration. Proc Natl Acad Sci USA 2002;99:14682-14687.

4. Friedlander M. Fibrosis and diseases of the eye. J Clin Invest 2007;117: 576-586.

5. Campochiaro PA, Soloway P, Ryan SJ et al. The pathogenesis of choroidal neovascularization in patients with age-related macular degeneration. Mol Vis 1999;5:34.

6. Jager RD, Mieler WF, Miller JW. Age-related macular degeneration. N Engl J Med 2008;358:2606-2617.

7. Lalwani GA, Rosenfeld PJ, Fung $A E$ et al. A variable-dosing regimen with intravitreal ranibizumab for neovascular age-related macular degeneration: year 2 of the PrONTO Study. Am J Ophthalmol 2009;148: 43-58.

8. Holz FG, Amoaku W, Donate J et al. Safety and efficacy of a flexible dosing regimen of ranibizumab in neovascular age-related macular degeneration: the SUSTAIN study. Ophthalmology 2011;118:663-671.

9. Daniel E, Toth CA, Grunwald JE et al. Risk of scar in the comparison of age-related macular degeneration treatments trials. Ophthalmology 2014;121:656-666.

10. Hans E, Grossniklaus HE, Kang SJ et al. Animal models of choroidal and retinal neovascularization. Progr Retin Eye Res 2010;29:500-519.

11. Lambert V, Lecomte J, Hansen S et al. Laser-induced choroidal neovascularization model to study age-related macular degeneration in mice. Nat Protocol 2013;8:2197-2211.

12. Tsutumi C, Sonoda $\mathrm{KH}$, Egashira $\mathrm{K}$ et al. The critical role of ocularinfiltrating macrophages in the development of choroidal neovascularization. J Leukoc Biol 2003;74:25-32.

13. Klein RJ, Zeiss C, Chew EY et al. Complement factor H polymorphism in age-related macular degeneration. Science 2005;308:385-389.

14. Edwards $\mathrm{AO}$, Ritter $\mathrm{R} 3 \mathrm{rd}$, Abel $\mathrm{KJ}$ et al. Complement factor $\mathrm{H}$ polymorphism and age-related macular degeneration. Science 2005;308:421-424.

15. Haines JL, Hauser MA, Schmidt S et al. Complement factor $\mathrm{H}$ variant increases the risk of age-related macular degeneration. Science 2005;308:419-421.

16. Nozaki M, Raisler BJ, Sakurai E et al. Drusen complement components C3a and C5a promote choroidal neovascularization. Proc Natl Acad Sci USA 2006;103:2328-2333.

17. Yoshida T, Ohno-Matsui $K$, Ichinose $S$ et al. The potential role of amyloid beta in the pathogenesis of age-related macular degeneration. J Clin Invest 2005;115:2793-2800.

18. Imamura $Y$, Noda S, Hashizume $K$ et al. Drusen, choroidal neovascularization, and retinal pigment epithelium dysfunction in SOD1-deficient mice: a model of age-related macular degeneration. Proc Natl Acad Sci USA 2006;103:11282-11287.

19. Ding $X$, Patel M, Chan CC. Molecular pathology of age-related macular degeneration. Prog Retin Eye Res 2009;28:1-18.

20. Naldini A, Carraro F. Role of inflammatory mediators in angiogenesis. Curr Drug Targets Inflamm Allerg 2005;4:3-8.

21. Nussenblatt RB, Ferris F. Age-related macular degeneration and the immune response: implications for therapy. Am J Ophthalmol 2007; 144:618-626.

22. Yasukawa T. Inflammation in age-related macular degeneration: pathological or physiological? Expert Rev Ophthalmol 2009;4: 107-112.

23. Grindle CF, Marshall J. Ageing changes in Bruch's membrane and their functional implications. Trans Ophthalmol Soc UK 1978;98: 172-175.

24. Oh H, Takagi $\mathrm{H}$, Takagi $\mathrm{C}$ et al. The potential angiogenic role of macrophages in the formation of choroidal neovascular membranes. Invest Ophthalmol Vis Sci 1999;40:1891-1898.

25. Tsutsumi C, Sonoda KH, Egashira K et al. The critical role of ocularinfiltrating macrophages in the development of choroidal neovascularization. J Leukoc Biol 2003;74:25-32.

26. Grossniklaus HE, Ling JX, Wallace TM et al. Macrophage and retinal pigment epithelium expression of angiogenic cytokines in choroidal neovascularization. Mol Vis 2002;8:119-126.

27. Killingsworth MC, Sarks JP, Sarks SH. Macrophages related to Bruch's membrane in age-related macular degeneration. Eye 1990;4: 613-621.

28. Espinosa-Heidmann DG, Suner IJ, Hernandez EP et al. Macrophage depletion diminishes lesion size and severity in experimental choroidal neovascularization. Invest Ophthalmol Vis Sci 2003;44: 3586-3592.

29. Sakurai E, Anand A, Ambati BK et al. Macrophage depletion inhibits experimental choroidal neovascularization. Invest Ophthalmol Vis Sci 2003;44:3578-3585.

30. Du H, Sun X, Guma M et al. JNK inhibition reduces apoptosis and neovascularization in a murine model of age-related macular degeneration. Proc Natl Acad Sci USA 2013;110:2377-2382.

31. Sumioka T, Ikeda K, Okada $Y$ et al. Inhibitory effect of blocking TGFbeta/Smad signal on injury-induced fibrosis of corneal endothelium. Mol Vis 2008;14:2272-2281.

32. Saika S, Kono-Saika S, Ohnishi $Y$ et al. Smad3 signaling is required for epithelial-mesenchymal transition of lens epithelium after injury. Am J Pathol 2004;164:651-663.

33. Saika S, Kono-Saika S, Tanaka T et al. Smad3 is required for dedifferentiation of retinal pigment epithelium following retinal detachment in mice. Lab Invest 2004;84:1245-1258.

34. Saika S, Yamanaka O, Nishikawa-Ishida I et al. Effect of Smad7 gene overexpression on transforming growth factor beta-induced retinal pigment fibrosis in a proliferative vitreoretinopathy mouse model. Arch Ophthalmol 2007;125:647-654.

35. Saika S, Ikeda K, Yamanaka O et al. Expression of Smad7 in mouse eyes accelerates healing of corneal tissue after exposure to alkali. Am J Pathol 2005;166:1405-1418.

36. Saika S, lkeda K, Yamanaka O et al. Transient adenoviral gene transfer of Smad7 prevents injury-induced epithelial-mesenchymal transition of lens epithelium in mice. Lab Invest 2004;84:1259-1270.

37. Goumans MJ, Valdimarsdottir G, Itoh $S$ et al. Balancing the activation state of the endothelium via two distinct TGF- $\beta$ type I receptors. EMBO J 2002;21:1743-1753.

38. Fujita N, Fujita S, Ogata N et al. Endogeenous osteopontin involvement in laser-induced choroidal neovascularization in mice. Invest Ophthalmol Vis Sci 2011;52:9310-9315.

39. Fujita S, Saika S, Kao WW et al. Endogenous TNFalpha suppression of neovascularization in corneal stroma in mice. Invest Ophthalmol Vis Sci 2007;48:3051-3055.

40. Kitano A, Saika S, Yamanaka O et al. Emodin suppression of ocular surface inflammatory reaction. Invest Ophthalmol Vis Sci 2007;48: 5013-5022.

41. Jinnin $M$, Ihn $H$, Tamaki K. Characterization of SIS3, a novel specific inhibitor of Smad3, and its effect on transforming growth factor-b1induced extracellular matrix expression. Mol Pharmacol 2006;69: 597-607.

42. Laping NJ, Grygielko E, Mathur A et al. Inhibition of transforming growth factor (TGF)- $\beta 1$-induced extracellular matrix with a novel inhibitor of the TGF- $\beta$ type I receptor kinase activity: SB-431542. Mol Pharmacol 2002;62:58-64.

43. Saika S, Ikeda S, Yamanaka $\mathrm{O}$ et al. Expression of Smad7 in mouse eyes accelerates healing of corneal tissue after exposure to alkali. Am J Pathol 2005;166:1405-1418.

44. Van Geest RJ, Lesnik-Oberstein SY, Tan HS et al. A shift in the balance of vascular endothelial growth factor and connective tissue growth factor by bevacizumab causes the angiofibrotic switch in proliferative diabetic retinopathy. Br J Opthalmol 2012;96:587-590.

45. Hwang JC, Del Priore LV, Freund KB et al. Development of subretinal fibrosis after anti-VEGF treatment in neovascular age-related macular degeneration. Ophthalmic Surg Lasers Imaging 2011;42:6-11.

46. Bloch SB, Lund-Andersen H, Sander B et al. Subfoveal fibrosis in eyes with neovascular age-related macular degeneration treated with intravitreal ranibizumab. Am J Ophthalmol 2013;156:116-124.

47. Ishikawa K, Kannan R, Hinton DR. Molecular mechanisms of subretinal fibrosis in age-related macular degeneration. Exp Eye Res 2016;142: 19-25.

48. Jo YJ, Sonoda KH, Oshima $Y$ et al. Establishment of a new animal model of focal subretinal fibrosis that resembles disciform lesion in advanced age-related macular degeneration. Invest Ophthalmol Vis Sci 2011;52: 6089-6095.

49. Zandi S, Nakao S, Chun KH et al. ROCK-isoform-specific polarization of macrophages associated with age-related macular degeneration. Cell Rep 2015;10:1173-1186.

50. Hu W, Jiang $Z$, Zhang $Y$ et al. Characterization of infiltrating macrophages in high glucose-induced peritoneal fibrosis in rats. Mol Med Rep 2012;6:93-99. 
51. Gong $D$, Shi $W$, Yi SJ et al. TGF $\beta$ signaling plays a critical role in promoting alternative macrophage activation. BMC Immunol 2012; 13:31.

52. Whitman M, Raftery L. TGFbeta signaling at the summit. Development 2005;132:4205-4210.

53. Goumans $\mathrm{MJ}$, Valdimarsdottir $\mathrm{G}$, Itoh $\mathrm{S}$ et al. Activin receptor-like kinase (ALK) 1 is an antagonistic mediator of lateral TGFbeta/ALK5 signaling. Mol Cell 2003;12:817-828.

54. Ota T, Fujii M, Sugizaki T et al. Targets of transcriptional regulation by two distinct type I receptors for transforming growth factor-beta in human umbilical vein endothelial cells. J Cell Physiol 2002;193: 299-318.
55. Nakagawa T, Li JH, Garcia G et al. TGF-beta induces proangiogenic and antiangiogenic factors via parallel but distinct Smad pathwaysKidney Int 2004;66:605-613.

56. Tian H, Mythreye K, Golzio $C$ et al. Endoglin mediates fibronectin/a5 $\beta 1$ integrin and TGF- $\beta$ pathway crosstalk in endothelial cells. EMBO J 2012;31:3885-3900.

57. Yang B, Cao DJ, Sainz I et al. Different roles of ERK and p38 MAP kinases during tube formation from endothelial cells cultured in 3-dimensional collagen matrices. Cell Physiol 2004;200:360-369.

58. Wang $\mathrm{S}, \mathrm{Cao} \mathrm{W}, \mathrm{Xing} \mathrm{H}$ et al. Activation of ERK pathway is required for 15-HETE-induced angiogenesis in human umbilical vascular endothelial cells. J Recept Signal Transduct Res 2015;13:1-8. 